\title{
Non-Wear Time and Presentation of Compositional 24-Hour Time-Use Analyses Influence Conclusions About Sleep and Body Mass Index in Children
}

\author{
Jillian J. Haszard, Kim Meredith-Jones, Victoria Farmer, Sheila Williams, \\ Barbara Galland, and Rachael Taylor \\ University of Otago
}

\begin{abstract}
Although 24-hour time-use data are increasingly being examined in relation to indices of health, consensus has yet to be reached about the best way to present estimates from compositional analyses. This analysis explored the impact of different presentations of results when assessing the relationship between 24-hour time-use and body mass index (BMI) $z$-score using compositional analysis of 5-day actigraphy data in 742 children. First it was found that reallocating non-wear time to day-time components only (sedentary behavior, light physical activity, and moderate-to-vigorous physical activity [MVPA]) before normalization to 24 hours provided stronger estimates with BMI $z$-score than simply removing non-wear time before normalization. Estimates for sleep time were substantially affected, where associations with BMI $z$-score nearly doubled (mean difference [95\% CI] in BMI $z$-score for $10 \%$ longer sleep were $-0.20[-0.32,-0.08]$ compared to $-0.11[-0.23,0.002])$. Presenting estimates in terms of a greater number of minutes in a component, relative to all others, showed MVPA to be the strongest predictor of BMI $z$-score, while estimates in terms of the proportion of minutes showed sleep to be the strongest predictor. Both presentations have value. However, presentations in terms of one-to-one "substitutions" of time may need careful interpretation due to the uneven distribution of time in each component. In conclusion, when analyzing relationships between 24-hour time-use and health outcomes, non-wear time and presentation of estimates can impact final conclusions. As a result, the current understanding of the importance of sleep for child health may be underestimated.
\end{abstract}

Keywords: actigraphy, compositional data analysis, physical activity, sedentary behavior

Evidence suggests that both physical activity and sleep are beneficial for the healthy development of children (Chaput et al., 2016; Matricciani, Paquet, Galland, Short, \& Olds, 2019; Poitras et al., 2016) whereas sedentary behavior may be detrimental (Carson et al., 2016). However, as these three time-use variables all occur within the 24-hour day, they are not independent, requiring appropriate statistical analysis.

Different activities in a 24-hour period are generally classified into a number of components, which necessarily add to 24 hours and are thus interdependent and constrained. This type of data are referred to as compositional time-use data. Statistical approaches for compositional data were originally developed for use in geology by John Aitchison (Aitchison, 1982), and have been recently refined for time-use data by several research groups (Chastin, Palarea-Albaladejo, Dontje, \& Skelton, 2015; Dumuid, Stanford, Martin-Fernández, et al., 2018; Dumuid, Stanford, Pedišić, et al., 2018; Fairclough et al., 2017; Pedišić, Dumuid, \& Olds, 2017; Talarico \& Janssen, 2018; Williams, Farmer, Taylor, \& Taylor, 2014). While statistical methods for assessing relationships with 24-hour time-use data now appear well-developed, questions remain regarding how to best prepare these complex data prior to analysis and how to express estimates of the association with

Haszard and Williams are with the Centre for Biostatistics; Meredith-Jones and Taylor are with the Department of Medicine; Farmer is with the Department of Research and Enterprise; Galland is with the Department of Women's and Children's Health; University of Otago, Dunedin, New Zealand. Haszard (jill.haszard@ otago.ac.nz) is corresponding author. health outcomes of interest. As associations with compositional variables are always expressed relative to the rest of the composition, the proportion of the 24-hour day that one component takes can greatly influence the interpretation of the results. In free-living situations the components of the day are not evenly distributed; for example, moderate-to-vigorous physical activity (MVPA) is usually a very small proportion of the day, whereas total sleep time is a very large proportion of the day. As this uneven distribution can be overlooked when interpreting results from compositional analyses, a comprehensive understanding of the strength of association between time-use components and health outcomes may still be missing.

When preparing 24-hour accelerometry data for analysis the day must first be defined. While midnight to midnight periods can be used, if all components are to be kept intact the day must be determined as the period from sleep offset to sleep offset (thereby keeping the sleep component unbroken). This means that there will be variation in day length and component variables must be normalized to sum to 24 hours. As part of this process, nonwear time is generally removed from the day before normalization (Carson, Tremblay, \& Chastin, 2017; Chastin et al., 2015; Dumuid, Stanford, Martin-Fernández, et al., 2018; Talarico \& Janssen, 2018) which effectively reallocates time in the day when the accelerometer was not worn to all other behaviors proportionally, including sleep. In many studies it can be assumed that non-wear periods are unlikely to occur during the night (i.e., between sleep onset and sleep offset), because they either measure sleep by questionnaire (using accelerometry for wake-time) or exclude 
any days where it appears that the accelerometer was taken off in the night (like in this study). The process of removing non-wear time before normalization therefore inappropriately allocates some non-wear time to sleep. This is crucial given the importance of sleep for health (Buysse, 2014). What effect the introduction of misclassified sleep might have when assessing relationships with health is not known.

Estimates of the relationship between compositional time-use and health outcomes (such as body mass index [BMI]) have generally been presented in terms of a "reallocation" of time in terms of a set block of minutes (e.g., 15 minutes) (Carson et al., 2017; Chastin et al., 2015; Dumuid, Stanford, Pedišić, et al., 2018; Fairclough et al., 2017). However, this does not acknowledge the relative contribution of time from each component to the total 24 hours in a day. For example, as MVPA represents a much smaller proportion of the day than sleep, a 15-minute reallocation of MVPA represents a much larger change in MVPA than the corresponding change in sleep. Furthermore, if estimates of association are presented in terms of a one-to-one "substitution" of time spent in one behavior to time spent in another, as has typically been done previously (Carson et al., 2017; Chastin et al., 2015; Dumuid, Stanford, Pedišić, et al., 2018; Fairclough et al., 2017), conclusions may be different to those found using a substitution of time from one behavior to all others proportionally. While the different presentations of estimates are not wrong, and indeed are all just different representations from the same statistical model, how they affect associations with health outcomes is unknown.

Until recently, compositional data have been analyzed using isotemporal substitution analyses, where one component is left out of the model to account for collinearity (Mekary, Willett, Hu, \& Ding, 2009). However, this method does not account for the constrained and compositional nature of the data and new compositional methods, based on the use of isometric log-ratios, are now widely accepted. While there are theoretical limitations to the use of isotemporal substitution methods, the practical impact that true compositional methods make on results for the investigation of time-use and body composition in children has only been examined using one-to-one reallocations of time (Chastin et al., 2015; Dumuid, Stanford, Martin-Fernández, et al., 2018; Dumuid, Stanford, Pedišić, et al., 2018).

To illustrate the potential impact of these issues in preparing, presenting, and interpreting compositional data analysis, we have used actigraphy data from a large study of New Zealand children in relation to BMI. We demonstrate the effect on estimates of association with BMI $z$-score by method of reallocating non-wear time; presentation in terms of proportions instead of minutes; presentation in terms of a one-to-one reallocation of time; and finally, by analyzing using traditional isotemporal methods in terms of compositions that differ by a proportion. We finish with suggestions for the preparation of data and reporting of estimates for a compositional data analysis of 24-hour time-use.

\section{Methods}

\section{Participants}

The data for these analyses are from the baseline assessment of a cluster-randomized controlled trial examining the effect of a playground intervention on physical activity and weight in primary school-aged children (the PLAY study) (Farmer et al., 2017). Participants were recruited from primary schools in Otago $(n=8)$ and Auckland $(n=8)$ in New Zealand. All children in school years
2 and 4 were invited to participate in the assessments and signed consent was obtained from parents. Baseline data were collected between March and December 2011. Ethical approval was obtained from the University of Otago (reference 10/077) and Auckland University of Technology (reference 10/95) Ethics Committees.

\section{BMI z-Score}

Height and weight were measured in duplicate using standard protocols. BMI was calculated and $z$-scores determined using the World Health Organization reference data (de Onis, Onyango, Van den Broeck, Chumlea, \& Martorell, 2004).

\section{Accelerometry}

Accelerometers (Actigraph GT3X, Actigraph Corp, Pensacola, FL, USA) were worn over the right hip 24 hours a day for 7 days, except for water-based activities such as bathing. Accelerometers were initialized using ActiLife (version 6, Actigraph Corp., Pensacola, FL, USA) in uniaxial mode using 15-s epochs. Data were cleaned and scored using an automated script developed in MATLAB (MathWorks, Natick, MA, USA) that removes the appropriate sleep period for each day for each child individually, to avoid sleep being misclassified as sedentary time (MeredithJones, Williams, Galland, Kennedy, \& Taylor, 2016). A day was considered valid if there were at least ten valid awake hours and if the total time (from time awake on one day to time awake the next day) was between 20 and 28 hours. Non-wear time (awake hours only) was defined as at least 20 min of consecutive zeros. Participants were excluded from the analysis if fewer than three valid days of wear were obtained. Activity intensities were calculated using the Evenson cut-points developed for children aged 5-8 years (Evenson, Catellier, Gill, Ondrak, \& McMurray, 2008).

\section{Statistical Analysis}

Statistical analysis was undertaken in Stata 15.1 (StataCorp, College Station, TX, USA). All analysis accounted for school clusters by using a mixed effects regression model with school as a random effect. BMI $z$-score was the outcome variable and the timeuse variables were the exposure variables. To illustrate more clearly the impact of the different preparation and presentation methods on the relationship between compositional data and BMI $z$-score, associations were not adjusted for any demographic covariates. To demonstrate the effect of different ways of treating missing data and/or reporting methods, estimates of association with BMI $z$-score were compared to a 'base model'. Regression coefficients, 95\% confidence intervals (CI), and $p$-values were calculated and reported as described in subsequent paragraphs.

The base model used for comparison included four time-use variables (components): sleep, sedentary, light physical activity (LPA), and MVPA; and was analyzed using compositional data analysis methods. Time spent awake after sleep onset was included in 'sleep time', as well as naps. Non-wear time was reallocated to other day-time components (sedentary, LPA, and MVPA) by multiplying the proportions of day-time wear by total day-time in minutes (including non-wear time). As each day was measured from wake-time to wake-time (to capture a full night's sleep) the total time could vary between 20 to 28 hours. Therefore the proportion of time spent in each component was multiplied by 1440 minutes ( 24 hours) to normalize the data to 24 hours. Means for all valid days were calculated weighted for weekends. 
The statistical analysis of compositional data using isometric log ratios is described in detail elsewhere (Chastin et al., 2015; Dumuid, Stanford, Martin-Fernández, et al., 2018). Briefly, isometric log ratios were calculated for each component. Each component had a set of three isometric log ratio coordinates, with the first coordinate being proportional to the log ratio of the component of interest to the geometric mean of the remaining components. All three coordinates were included in the mixed effects regression model for the component of interest (e.g., sleep) and the regression coefficient of the first coordinate described the strength of the relationship between the component of interest (e.g., sleep) relative to the remaining components and BMI $z$-score. To interpret the strength of association, the regression coefficient was further transformed using the method described by Dumuid, Stanford, Martin-Fernández, et al. (2018). Thus, the associations were reported as the difference in BMI $z$-score for a time-use composition that is $10 \%$ greater (than average) in the time spent in that component, reallocated proportionally from all other time-use components.

\section{Removing Non-Wear Time}

Rather than simply removing non-wear time before normalization to 24-hours, we reallocated non-wear time to other day-time components (sedentary, LPA, MVPA) proportionally on the basis of time they contributed to the total day. The difference between these reallocation methods (removing it from the total composition compared to reallocating it to only day-time components) is illustrated visually using one participant's data in Figure 1. To compare this handling of non-wear time to simply removing it (in effect reallocating in proportionally to all components including sleep), the base model was run on both sets of data and associations with BMI $z$-score were reported for both.

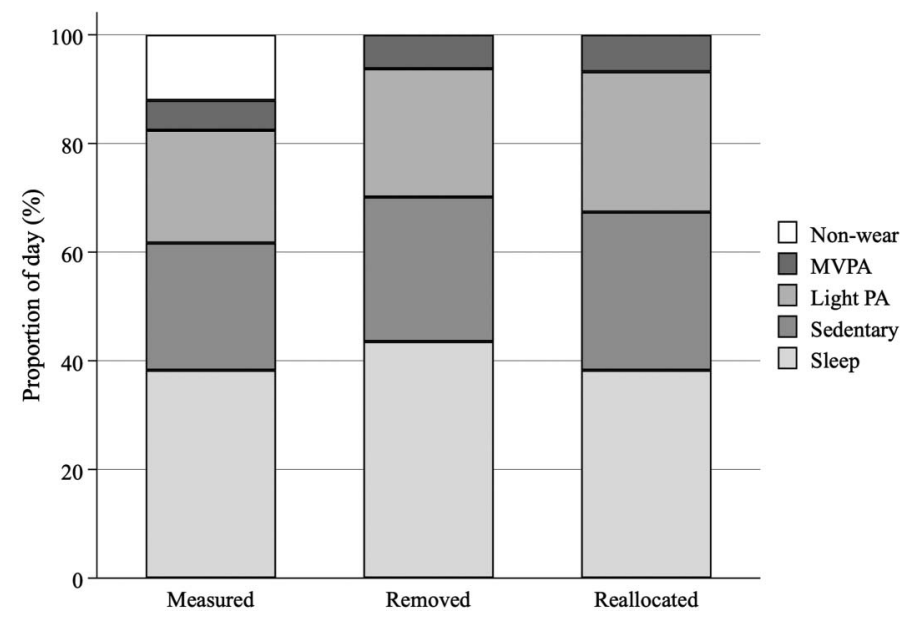

Figure 1 - Illustration of how non-wear time can influence the composition of the 24-hour day using one participant's data. Non-wear time during the day is missing data but represents a proportion of time (Measured). If it is removed before normalization (as is the standard approach in current research) that time gets reallocated to all other behaviors, proportionally (Removed). However, non-wear time does not occur during the night and when reallocated to day-time activities only (Reallocated) time spent asleep is not artificially inflated (as seen in Removed).

\section{Reporting Estimates as a Reallocation of Time in Minutes}

To investigate how presentation of the associations with BMI $z$-score in terms of minutes instead of proportions affected the interpretation of the results, the base model was run but the regression coefficients reported in terms of a 10-minute greater time spent in the component of interest, reallocated proportionally from all other time-use components.

\section{Reporting Estimates as a One-to-One Reallocation of Time From One Component to Another Component}

The presentation of estimates of association with BMI $z$-score for a composition that differs by only two components (e.g., an increase in sleep and decrease in MVPA, while the rest of the composition remains the same) was calculated using the base model. The normalized geometric means were calculated for the mean composition of time and for the new composition, adding 10\% (or 10 minutes) to one component (e.g., MVPA) with the equivalent time (\% or minutes) taken from another component (e.g., sleep). The estimated difference in BMI $z$-score associated with this difference in composition was determined by substituting the mean composition into the model and then substituting the new composition into the model, and subtracting these as per the method described in (Fairclough et al., 2017). Estimates were calculated for both a $10 \%$ reallocation of time and a 10-minute reallocation of time.

\section{Traditional Isotemporal Analysis}

Finally, to illustrate the difference that appropriate compositional analysis makes compared to the traditional isotemporal analysis, this analysis was also undertaken. Data was first normalized to 24 hours after reallocation of non-wear time to day-time components. Traditional isotemporal analysis has the minutes of each component as the predictor variables in the model but leaves out the comparison component to account for the fact that they all add to 24 hours (Colley, Michaud, \& Garriguet, 2018; Mekary et al., 2009). After the model was generated, the difference in estimated BMI $z$-score (and 95\% CI) was calculated for mean time-use composition compared to the mean time-use composition with time reallocated as specified. Estimates were determined using the 'nlcom' in Stata and presented in terms of a $10 \%$ reallocation for comparison to the base model.

\section{Results}

Seven hundred and forty-two participants from the PLAY study had complete accelerometry data and a BMI $z$-score measure. Demographic and other characteristics of this sample are described in Table 1. On average they had 5.2 days of valid data, ranging from 3 to 6 valid days. They spent a mean $41 \%$ of their 24 hours asleep, $30 \%$ sedentary, $21 \%$ in LPA, and 5\% in MVPA. On average, participants had $3 \%$ non-wear time.

\section{Removing Non-Wear Time}

Table 2 illustrates that the different ways of reallocating non-wear time substantially influenced the estimates of association with BMI $z$-score, with nearly all associations being weaker when non-wear 
Table 1 Characteristics of the Study Population $(n=742)$

\begin{tabular}{|c|c|}
\hline & $n(\%)^{a}$ \\
\hline Sex (female) & $381(51.4)$ \\
\hline \multicolumn{2}{|l|}{ Residential area deprivation ${ }^{\mathrm{b}}$} \\
\hline Low $(1-3)$ & $103(17.1)$ \\
\hline Medium (4-7) & $290(48.2)$ \\
\hline High $(8-10)$ & $209(34.7)$ \\
\hline Age (years), $M(S D)$ & $8.0(1.1)$ \\
\hline \multicolumn{2}{|l|}{ Ethnicity ${ }^{\mathrm{b}}$} \\
\hline European & $397(57.6)$ \\
\hline Māori & $140(20.3)$ \\
\hline Pacific Island & $92(13.4)$ \\
\hline Asian & $60(8.1)$ \\
\hline BMI z-score, $M(S D)$ & $0.69(1.07)$ \\
\hline \multicolumn{2}{|l|}{ Weight status } \\
\hline Normal weight & $492(66.3)$ \\
\hline Overweight & $130(17.5)$ \\
\hline Obese & $120(16.2)$ \\
\hline Number days valid data, $M(S D)$ & $5.2(0.8)$ \\
\hline Non-wear time (minutes), $M(S D)$ & $42(32)$ \\
\hline Sleep time (minutes), $M(S D)$ & $591(36)$ \\
\hline Sedentary time (minutes), $M(S D)$ & $433(57)$ \\
\hline Light physical activity (minutes), $M(S D)$ & 307 (44) \\
\hline Moderate-to-vigorous physical activity (minutes), $M(S D)$ & $67(23)$ \\
\hline
\end{tabular}

Note. $M(S D)=$ mean (standard deviation).

${ }^{\mathrm{a}}$ unless otherwise indicated. ${ }^{\mathrm{b}} n=140$ were missing deprivation data; $n=44$ did not have ethnicity data or identified as 'other' $(n=9)$.

time is simply removed before normalization. This was most marked for the association between sleep and BMI $z$-score, which nearly doubled when non-wear time was not allocated to sleep.

\section{Reporting Estimates as a Reallocation of Time in Both Percentage Terms and Minutes}

Table 3 first reports the estimates of association with BMI $z$-score using the regression coefficients for the first isometric log ratio coordinate from the base model. This shows that although the direction of association (i.e., positive or negative) with BMI $z$-score is easy to interpret, the strength of the association is more difficult. The 4th column reports the difference in BMI $z$-score obtained by reallocating $10 \%$ of the mean time of the component of interest to the component of interest, from all remaining components, proportionally. For example, $10 \%$ of sleep was, on average, 59 minutes, which was added to sleep time, and 5 minutes taken from MVPA, 32 minutes from sedentary, and 22 minutes from LPA to keep the total time to 24 hours. From here it can be seen that the strongest relationship is between BMI $z$-score and sleep. Reallocating $10 \%$ more time (on average 59 minutes) to sleep, with a reduction in time of all other components, was associated with a lower BMI $z$-score by 0.20 (0.08, 0.32). Conversely, allocating $10 \%$ more time to light physical activity from all other components was associated with a higher BMI $z$-score by $0.19(0.12,0.25)$. MVPA was also negatively associated with BMI $z$-score but not as strongly as sleep-with a $10 \%$ greater proportion of time spent in MVPA (with a corresponding reduction in time in all other components) related to a 0.07 ( 0.04 , 0.09) lower BMI $z$-score.

Also in Table 3, using the same model, the estimates of the associations were reported in terms of a change in composition by minutes (instead of proportion of time). This presentation of the results showed that the component with the strongest association with BMI $z$-score was now MVPA, where a composition with 10 minutes more allocated to MVPA was associated with a lower BMI $z$-score by $0.10(0.06,0.13)$. By contrast, allocating 10 more minutes to sleep was associated with a lower BMI $z$-score by only $0.04(0.01,0.05)$.

\section{Reporting Estimates as a One-to-One Reallocation of Time From One Component to Another Component}

Table 4 shows estimates of association with BMI $z$-score if the mean composition is compared to a composition where time has been reallocated from one component to just one other component, as opposed to reallocating proportionally across all other components. These estimates are presented using both $10 \%$ and 10-minute reallocation. These mixed findings are difficult to interpret. Inconsistency lies in the associations with sleep, where a reallocation from sedentary or LPA to sleep is beneficial as well as a reallocation of sleep to MVPA, meaning that any reallocation to sleep would seem better reallocated to MVPA. This leaves the relationship between sleep and BMI $z$-score equivocal.

Table 2 Relationships Between 24-Hour Time-Use and BMI z-Score Using Compositional Analysis ${ }^{a}$ of Data With Non-Wear Time Reallocated by Two Different Methods $(n=742)$

\begin{tabular}{|c|c|c|c|c|c|c|}
\hline \multirow[b]{2}{*}{ Component } & \multicolumn{3}{|c|}{ Non-Wear Time Reallocated to All Components } & \multicolumn{3}{|c|}{$\begin{array}{c}\text { Non-Wear Time Reallocated to Day-Time } \\
\text { Components }\end{array}$} \\
\hline & $\begin{array}{l}\text { Average } 10 \% \\
\text { of Component } \\
\text { in Minutes }\end{array}$ & $\begin{array}{c}\text { Estimated Difference }(95 \% \mathrm{Cl}) \text { in } \\
\text { BMI z-Score With a } 10 \% \\
\text { Reallocation of Time to } \\
\text { Component }^{\mathbf{b}}\end{array}$ & $\begin{array}{c}p- \\
\text { value }\end{array}$ & $\begin{array}{l}\text { Average } 10 \% \\
\text { of Component } \\
\text { in Minutes }\end{array}$ & $\begin{array}{l}\text { Estimated Difference } \\
(95 \% \mathrm{Cl}) \text { in BMI z-Score } \\
\text { With a } 10 \% \text { Reallocation of } \\
\text { Time to Component }\end{array}$ & $\begin{array}{c}p- \\
\text { Value }\end{array}$ \\
\hline Sleep & 61 & $-0.11(-0.23,0.002)$ & .055 & 59 & $-0.20(-0.32,-0.08)$ & .001 \\
\hline Sedentary & 44 & $0.02(-0.05,0.08)$ & 627 & 45 & $0.06(-0.01,0.12)$ & .111 \\
\hline LPA & 32 & $0.16(0.09,0.21)$ & $<.001$ & 32 & $0.19(0.12,0.25)$ & $<.001$ \\
\hline MVPA & 6.6 & $-0.07(-0.09,-0.05)$ & $<.001$ & 6.7 & $-0.07(-0.09,-0.04)$ & $<.001$ \\
\hline
\end{tabular}

Note. $95 \% \mathrm{CI}=95 \%$ confidence interval; $\mathrm{BMI}=$ body mass index; LPA = light physical activity; MVPA = moderate-to-vigorous physical activity.

${ }^{a}$ Compositional analysis involves a regression model using isometric log-ratios as predictors, calculated using a different permutation of the components for each component of interest. ${ }^{b}$ Time is reallocated from all other components proportionally to the component of interest. 
Table 3 Relationships Between 24-Hour Time-Use and BMI z-Score Using Compositional Analysis ${ }^{\mathrm{a}}$ and Presented Using Raw Regression Coefficients and Reallocations in Terms of Proportions and Minutes $(n=742)$

\begin{tabular}{|c|c|c|c|c|c|}
\hline Component & $\begin{array}{l}\text { Average } 10 \% \\
\text { of } \\
\text { Component } \\
\text { in Minutes }\end{array}$ & $\begin{array}{l}\text { Regression } \\
\text { Coefficient for First } \\
\text { Isometric Log Ratio } \\
(95 \% \mathrm{Cl}) \\
\end{array}$ & $\begin{array}{c}\text { Estimated Difference }(95 \% \mathrm{Cl}) \\
\text { in BMI z-Score With a } 10 \% \\
\text { Reallocation of Time to } \\
\text { Component }^{\text {b }}\end{array}$ & $\begin{array}{l}\text { Estimated Difference }(95 \% \mathrm{Cl}) \text { in } \\
\text { BMI z-Score With a } 10-\text { Minute } \\
\text { reallocation of Time to } \\
\text { Component }^{b}\end{array}$ & $\begin{array}{c}p- \\
\text { Value }^{c}\end{array}$ \\
\hline Sleep & 59 & $-1.4(-2.2,-0.6)$ & $-0.20(-0.32,-0.08)$ & $-0.04(-0.05,-0.01)$ & .001 \\
\hline Sedentary & 46 & $0.4(-0.1,1.0)$ & $0.06(-0.01,0.12)$ & $0.01(-0.003,0.03)$ & .111 \\
\hline LPA & 32 & $1.7(1.1,2.3)$ & $0.19(0.12,0.25)$ & $0.06(0.04,0.08)$ & $<.001$ \\
\hline MVPA & 7 & $-0.8(-1.0,-0.5)$ & $-0.07(-0.09,-0.04)$ & $-0.10(-0.13,-0.06)$ & $<.001$ \\
\hline
\end{tabular}

Note. $95 \% \mathrm{CI}=95 \%$ confidence interval; $\mathrm{BMI}=$ body mass index; LPA = light physical activity; MVPA = moderate-to-vigorous physical activity.

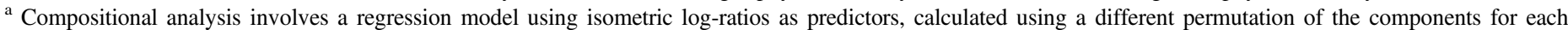
component of interest. ${ }^{\mathrm{b}}$ Time is reallocated from all other components proportionally to the component. ${ }^{\mathrm{c}} p$-value for the regression coefficient.

Table 4 Mean Differences (95\% Cl) Between 24-Hour Time-Use and BMI z-Score Using Compositional Analysis ${ }^{a}$ Presented as a Reallocation of Time Spent in One Component to One Other Component $(n=742)$

\begin{tabular}{|c|c|c|c|c|}
\hline Component & To Sleep & To Sedentary & To LPA & To MVPA \\
\hline \multicolumn{5}{|l|}{$10 \%$ reallocation } \\
\hline From sleep & & $0.17(0.05,0.30)$ & $0.38(0.24,0.51)$ & $-0.29(-0.47,-0.11)$ \\
\hline From sedentary & $-0.13(-0.22,-0.03)$ & & $0.15(0.07,0.23)$ & $-0.38(-0.49,-0.27)$ \\
\hline From LPA & $-0.22(-0.30,-0.14)$ & $-0.13(-0.19,-0.07)$ & & $-0.41(-0.53,-0.29)$ \\
\hline From MVPA & $0.06(0.03,0.09)$ & $0.08(0.05,0.10)$ & $0.10(0.07,0.13)$ & \\
\hline \multicolumn{5}{|c|}{ 10-minute reallocation } \\
\hline From sleep & & $0.03(0.01,0.05)$ & $0.07(0.04,0.09)$ & $-0.07(-0.11,-0.03)$ \\
\hline From sedentary & $-0.03(-0.05,-0.01)$ & & $0.04(0.02,0.05)$ & $-0.10(-0.13,-0.07)$ \\
\hline From LPA & $-0.07(-0.09,-0.04)$ & $-0.04(-0.06,-0.02)$ & & $-0.14(-0.18,-0.10)$ \\
\hline From MVPA & $0.09(0.04,0.13)$ & $0.11(0.08,0.15)$ & $0.15(0.11,0.20)$ & \\
\hline
\end{tabular}

Note . BMI = body mass index $; 95 \%$ CI = 95\% confidence interval; $\mathrm{LPA}=$ light physical activity; MVPA = moderate-to-vigorous physical activity.

${ }^{a}$ Compositional analysis involves a regression model using isometric log-ratios as predictors, calculated using a different permutation of the components for each component of interest.

Table 5 Relationships Between 24-Hour Time-Use and BMI z-Score Using Traditional Isotemporal Analyses and Compositional Analyses $(n=742)$

\begin{tabular}{|c|c|c|c|c|c|}
\hline \multirow[b]{2}{*}{ Component } & \multirow[b]{2}{*}{$\begin{array}{l}\text { Average } 10 \% \text { of } \\
\text { Component in } \\
\text { Minutes }\end{array}$} & \multicolumn{2}{|l|}{ Traditional Isotemporal Analysis $^{a}$} & \multicolumn{2}{|l|}{ Compositional Analysis $^{\text {b }}$} \\
\hline & & $\begin{array}{c}\text { Estimated Difference }(95 \% \mathrm{Cl}) \text { in } \\
\text { BMI z-Score With a } 10 \% \text { Increase in } \\
\text { Component }^{\mathrm{c}}\end{array}$ & $\begin{array}{c}p- \\
\text { value }\end{array}$ & $\begin{array}{c}\text { Estimated Difference }(95 \% \mathrm{Cl}) \text { in } \\
\text { BMI z-Score With a } 10 \% \text { Increase in } \\
\text { Component }^{c}\end{array}$ & $\begin{array}{c}p- \\
\text { Value }\end{array}$ \\
\hline Sleep & 59 & $-0.22(-0.34,-0.10)$ & $<.001$ & $-0.22(-0.35,-0.10)$ & .001 \\
\hline Sedentary & 45 & $0.07(0.001,0.14)$ & .046 & $0.07(-0.01,0.14)$ & .074 \\
\hline LPA & 32 & $0.17(0.11,0.23)$ & $<.001$ & $0.20(0.13,0.25)$ & $<.001$ \\
\hline MVPA & 7 & $-0.05(-0.07,-0.03)$ & $<.001$ & $-0.07(-0.09,-0.04)$ & $<.001$ \\
\hline
\end{tabular}

Note. $\mathrm{BMI}=$ body mass index; $95 \% \mathrm{CI}=95 \%$ confidence interval; $\mathrm{LPA}=$ light physical activity; $\mathrm{MVPA}=$ moderate-to-vigorous physical activity.

${ }^{\mathrm{a}}$ Traditional isotemporal analysis involves excluding the component of interest as a predictor in the regression model. ${ }^{\mathrm{b}}$ Compositional analysis involves a regression model using isometric log-ratios as predictors, calculated using a different permutation of the components for each component of interest. ${ }^{\mathrm{c}}$ Time is reallocated from all other components proportionally to the component of interest.

\section{Traditional Isotemporal Analysis}

Table 5 demonstrates that if traditional isotemporal methods of assessing the relationship between 24-hour time-use and BMI $z$-score are used, estimates of association are remarkably similar to results found using compositional analyses.

\section{Discussion}

We have shown that, in contrast to the use of traditional isotemporal statistical methods, which made little difference to estimates, compositional methods with less accurate reallocation of non-wear 
time and differing presentations of estimates had a greater influence on the associations between 24-hour time-use and BMI $z$-score. This was especially so for the relationship with sleep, the influence of which was largely underestimated by the compositional methods that are commonly practiced. In particular, the one-to-one substitution presentation of associations suggested that a longer sleep time (by decreasing MVPA) was related to higher BMI z-score, a result that was inconsistent with every other method of presentation. The implications for public health messages from these different approaches to compositional analysis are considerable, with inconsistencies adding confusion. Therefore, we present suggestions for the preparation and reporting of compositional analysis of 24-hour time-use data with health outcomes.

\section{Non-Wear Time Should be Reallocated as Accurately as Possible}

Non-wear time is essentially missing data, but it is usually measured as a proportion of the 24-hour day. However, non-wear time typically only occurs in day-time hours. This is because non-wear time at night will either be measured as sleep (if participants took the accelerometer off for sleep) or used as an exclusion criteria in studies where accurate measurement of sleep is prioritized (like this study). Previous investigations of compositional time-use and health outcomes have tended to simply remove non-wear time before normalization to 24-hours (Carson et al., 2017; Chastin et al., 2015; Dumuid, Stanford, Martin-Fernández, et al., 2018; Talarico \& Janssen, 2018). We have shown that compared to this method, reallocating non-wear time proportionally to only day-time activities before normalization strengthened the estimates of association with BMI $z$-score. This was most marked with sleep, where the estimate of association nearly doubled when non-wear time was not allocated to sleep time. This suggests that our current understanding of the relationship between sleep and health outcomes may be underestimated. While this method of reallocation of non-wear time is both sensible and simple, it illustrates the influence of non-wear time on results. However, this method is still an imputation of missing data, which will not always reflect the true time-use patterns of the participant. Therefore, it should be a priority for future studies to achieve 24-hour wear-time compliance.

\section{Relationships Between Health Outcomes and 24-Hour Time-Use Should Be Reported by Both Proportion of Time and a Set Number of Minutes}

When determining the components of the 24-hour day that were most strongly associated with BMI $z$-score, presenting the associations in terms of proportions or minutes gave different results: For a small change in proportion of time, sleep was the component most strongly associated with lower BMI $z$-score, however the biggest difference in BMI $z$-score for a 10-minute reallocation was for MVPA. Most previous research has presented estimates in terms of minutes, which makes sense as it is a standard, meaningful unit of the day. However, this process can make associations with the smallest proportions (e.g., MVPA) seem stronger than those with the largest proportions (e.g., sleep). Thus because 10 minutes is a large proportion of mean time in MVPA and a very small proportion of mean sleep time, it makes sense to also present associations in terms of a proportion of component time. This allows us to compare the effect of different components of the day on health outcomes using a comparable unit. Here we showed that sleep and light physical activity were most strongly associated with BMI $z$-score for a $10 \%$ reallocation of time and therefore changes in these components may have the biggest effect. However, for a 10 minute reallocation of time, MVPA may be the most effective way to decrease BMI $z$-score. Both presentations are useful to improve understanding of associations between time-use composition and BMI z-score.

\section{Reporting Estimates for a One-to-One Substitution of Time Requires Careful Interpretation}

If estimates of association are presented in terms of a one-to-one substitution of time spent in one behavior to time spent in another as has typically been done previously (Carson et al., 2017; Chastin et al., 2015; Dumuid, Stanford, Pedišić, et al., 2018; Fairclough et al., 2017), misleading conclusions are possible. For example, from our results (Table 4) we may be inclined to recommend that children replace sleep with MVPA to reduce BMI $z$-score. This presentation of associations contradicts the alternative presentation from the very same statistical model shown in Table 3, which indicated that the strongest protective association with BMI $z$-score is with more time spent asleep. These mixed messages come about because of the uneven distribution of time-in our sample $10 \mathrm{~min}$ utes represents only a $1.7 \%$ change in sleep but a $14 \%$ change in MVPA-so in shifting a set amount of time between MVPA (where a small amount is very meaningful) and sleep (where a small amount has little meaning), the effect of MVPA overshadows any effect of sleep. Conversely, if a meaningful amount of time according to sleep (for example 30 minutes or an hour) was reallocated to sleep from MVPA, this may represent an unreasonable change in MVPA, and indeed may exceed the average time in MVPA. This is a key problem with using one-to-one reallocations interpretation of the results is susceptible to the imbalance in time across the day. In addition, the estimates from Table 4 assume that, apart from the two components involved in the substitution, the rest of the composition remains constant. This represents an artificial situation where sleep and MVPA can be interchanged with no effect on light activity or sedentary behavior. While this type of presentation might be useful in more controlled environments (such as replacing sitting with standing in a work-day), in freeliving situations interchanging two components without impacting other components seems unrealistic. Indeed, these analyses do not present findings that are easily translatable to public health messages. Instead, more appropriate recommendations to make from this data would be to increase sleep and MVPA at the expense of light activity and sedentary behaviors for BMI outcomes. These can be drawn from the results presented in Table 3 and do not need the inconsistent and unhelpful set of results drawn from the one-toone substitution presentation in Table 4.

\section{Traditional Isotemporal Approaches May Still be Valid}

While theoretically the traditional isotemporal methods for analyzing 24-hour time-use are not appropriate and compositional data analysis is recommended, in practice using compositional methodology made little difference to the results. It is difficult to compare our findings to others who have presented a comparison between these two methods in children, as they have generally used the one-to-one substitution (Dumuid, Stanford, Martin-Fernández, et al., 2018; Dumuid, Stanford, Pedišić, et al., 2018) or not used the same units for both methods (Chastin et al., 2015). Indeed, we found that non-wear time and the presentation of results from 
compositional analyses had a much greater effect on conclusions in our example, compared to using traditional isotemporal analyses. The use of compositional analyses also comes with further complications in the presence of zero values, which is not covered in detail here. Because compositional analysis uses log-ratios, zero values cannot be included, and must be combined into another component or imputed with a small number. The latter assumes that all zeros are rounded, which may not be the case, and there is no agreed-upon compositional method for handling true zeros. This limits the appropriate investigation of important components of the 24-hour day, such as time spent awake after sleep onset, or vigorous activity, which may have a strong influence on health outcomes but, as they often contain zero values, their inclusion in compositional analyses is compromised.

In conclusion, when investigating the strength of associations of sleep, sedentary behavior, and physical activity with health outcomes, care must be taken in the preparation of this compositional data and with the presentation of results, which may be used to inform the development of guidelines and interventions. The interpretation of relationships with compositional data is not straightforward. The units that the estimates are presented in $(\%$ or minutes, or how time is reallocated) can create major differences to the meaning of the results when using compositional data.

\section{References}

Aitchison, J. (1982). The statistical analysis of compositional data. Journal of the Royal Statistical Society. Series B (Methodological), 44(2), 139-177. doi:10.1111/j.2517-6161.1982.tb01195.x

Buysse, D.J. (2014). Sleep health: Can we define it? Does it matter? Sleep, 37(1), 9-17. PubMed ID: 24470692 doi:10.5665/sleep.3298

Carson, V., Hunter, S., Kuzik, N., Gray, C.E., Poitras, V.J., Chaput, J.-P., . . Tremblay, M.S. (2016). Systematic review of sedentary behaviour and health indicators in school-aged children and youth: An update. Applied Physiology, Nutrition, and Metabolism, 41(6 Suppl. 3), S240-S265. PubMed ID: 27306432 doi:10.1139/apnm-2015-0630

Carson, V., Tremblay, M.S., \& Chastin, S.F.M. (2017). Cross-sectional associations between sleep duration, sedentary time, physical activity, and adiposity indicators among Canadian preschool-aged children using compositional analyses. BMC Public Health, 17(5), 848. doi:10.1186/s12889-017-4852-0

Chaput, J.-P., Gray, C.E., Poitras, V.J., Carson, V., Gruber, R., Olds, T., ... Tremblay, M.S. (2016). Systematic review of the relationships between sleep duration and health indicators in school-aged children and youth. Applied Physiology, Nutrition, and Metabolism, 41(6 Suppl. 3), S266-S282. PubMed ID: 27306433 doi:10.1139/apnm2015-0627

Chastin, S.F.M., Palarea-Albaladejo, J., Dontje, M.L., \& Skelton, D.A. (2015). Combined effects of time spent in physical activity, sedentary behaviors and sleep on obesity and cardio-metabolic health markers: A novel compositional data analysis approach. PLoS ONE, 10(10), e0139984. PubMed ID: 26461112 doi:10.1371/journal.pone. 0139984

Colley, R.C., Michaud, I., \& Garriguet, D. (2018). Reallocating time between sleep, sedentary and active behaviours: Associations with obesity and health in Canadian adults. Health Reports, 29(4), 3-13. PubMed ID: 29668028.

de Onis, M., Onyango, A.W., Van den Broeck, J., Chumlea, C.W., \& Martorell, R. (2004). Measurement and standardization protocols for anthropometry used in the construction of a new international growth reference. Food and Nutrition Bulletin, 25(1 Suppl. 1), S27-S36. doi:10.1177/15648265040251S104

Dumuid, D., Stanford, T.E., Martin-Fernández, J.-A., Pedišić, Ž., Maher, C.A., Lewis, L.K., . . . Olds, T. (2018). Compositional data analysis for physical activity, sedentary time and sleep research. Statistical Methods in Medical Research, 27(12), 3726-3738. doi:10.1177/ 0962280217710835

Dumuid, D., Stanford, T.E., Pedišić, Ž., Maher, C., Lewis, L.K., MartínFernández, J.-A., ... Olds, T. (2018). Adiposity and the isotemporal substitution of physical activity, sedentary time and sleep among school-aged children: a compositional data analysis approach. BMC Public Health, 18(1), 311. PubMed ID: 29499689 doi:10.1186/ s12889-018-5207-1

Evenson, K.R., Catellier, D.J., Gill, K., Ondrak, K.S., \& McMurray, R.G. (2008). Calibration of two objective measures of physical activity for children. Journal of Sports Sciences, 26(14), 1557-1565. PubMed ID: 18949660 doi:10.1080/02640410802334196

Fairclough, S.J., Dumuid, D., Taylor, S., Curry, W., McGrane, B., Stratton, G., ... Olds, T. (2017). Fitness, fatness and the reallocation of time between children's daily movement behaviours: an analysis of compositional data. International Journal of Behavioral Nutrition and Physical Activity, 14(1), 64. PubMed ID: 28486972 doi:10.1186/ s12966-017-0521-z

Farmer, V.L., Williams, S.M., Mann, J.I., Schofield, G., McPhee, J.C., \& Taylor, R.W. (2017). The effect of increasing risk and challenge in the school playground on physical activity and weight in children: a cluster randomised controlled trial (PLAY). International Journal of Obesity, 41, 793-800. PubMed ID: 28186099 doi:10.1038/ijo. 2017.41

Matricciani, L., Paquet, C., Galland, B., Short, M., \& Olds, T. (2019). Children's sleep and health: A meta-review. Sleep Medicine Reviews, 46, 136-150. doi:10.1016/j.smrv.2019.04.011

Mekary, R.A., Willett, W.C., Hu, F.B., \& Ding, E.L. (2009). Isotemporal substitution paradigm for physical activity epidemiology and weight change. American Journal of Epidemiology, 170(4), 519-527. PubMed ID: 19584129 doi:10.1093/aje/kwp163

Meredith-Jones, K., Williams, S., Galland, B., Kennedy, G., \& Taylor, R. (2016). 24 h Accelerometry: impact of sleep-screening methods on estimates of sedentary behaviour and physical activity while awake. Journal of Sports Sciences, 34(7), 679-685. PubMed ID: 26194337 doi:10.1080/02640414.2015.1068438

Pedišić, Ž., Dumuid, D., \& Olds, T.S. (2017). Integrating sleep, sedentary behaviour, and physical activity research in the emerging field of time-use epidemiology: Definitions, concepts, statistical methods, theoretical framework, and future directions. Kinesiology, 49(2), 252-269.

Poitras, V.J., Gray, C.E., Borghese, M.M., Carson, V., Chaput, J.-P., Janssen, I., ... Tremblay, M.S. (2016). Systematic review of the relationships between objectively measured physical activity and health indicators in school-aged children and youth. Applied Physiology, Nutrition, and Metabolism, 41(6 Suppl. 3), S197-S239. PubMed ID: 27306431 doi:10.1139/apnm-2015-0663

Talarico, R., \& Janssen, I. (2018). Compositional associations of time spent in sleep, sedentary behavior and physical activity with obesity measures in children. International Journal of Obesity, 42(8), 1508-1514. PubMed ID: 29568110 doi:10.1038/s41366018-0053-x

Williams, S.M., Farmer, V.L., Taylor, B.J., \& Taylor, R.W. (2014). Do more active children sleep more? A repeated cross-sectional analysis using accelerometry. PLOS ONE, 9(4), e93117-e93117. PubMed ID: 24695112 doi:10.1371/journal.pone.0093117 